\title{
A FACTORIZATION OF THE DIRECT LIMIT OF HILBERT CUBES
}

\author{
RICHARD E. HEISEY
}

\begin{abstract}
We show that the countable direct limit of Hilbert cubes $Q^{\infty}$ is homeomorphic to the product of the Hilbert cube $Q$ and the countable direct limit of lines $R^{\infty}$. As a consequence, two open subsets of $R^{\infty}$ have the same homotopy type if and only if their products with $Q$ are homeomorphic. Combined with a theorem of D. W. Henderson our result implies that $X \times Q \times R^{\infty} \cong Q \times R^{\infty}$, where $X$ is any locally compact, separable $\mathrm{AR}$ (metric).
\end{abstract}

At the problem session at the Conference on Infinite-Dimensional Topology at Louisiana State University, October 1973, Tom Chapman asked if $R^{\infty} \times Q$ $\cong Q^{\infty}$. Here $R^{\infty}=\operatorname{dir} \lim R^{n}, Q^{\infty}=\operatorname{dir} \lim Q^{n}$, where $R$ denotes the reals and $Q$ denotes the Hilbert cube, which we regard as the countably infinite product of $I=[0,1]$. (We remark that if $B$ is any separable, infinitedimensional Banach space, then its conjugate $B^{*}$ with the bounded weak-* topology is homeomorphic to $Q^{\infty}$ [4, Theorem II-6].) We answer the above question in the affirmative. As a corollary we show (Corollary 5) that two open subsets of $R^{\infty}$ have the same homotopy type iff their products with $Q$ are homeomorphic. Finally, combining our result with work of D. W. Henderson [6] we obtain a factorization result for locally compact, separable AR's and ANR's (Theorem 7).

The author wishes to thank James West for several helpful conversations.

Let $J=[-1,1]$. Define $i_{n}: I^{n} \rightarrow I^{n+1}$ and $j_{n}: J^{n} \rightarrow J^{n+1}$ by $i_{n}\left(x_{1}, \ldots, x_{n}\right)$ $=\left(x_{1}, \ldots, x_{n}, 0\right), j_{n}\left(x_{1}, \ldots, x_{n}\right)=\left(x_{1}, \ldots, x_{n}, 0\right)$. Note that $i_{n}\left(I^{n}\right)$ is a $Z$-set in $I^{n+1}$, whereas $j_{n}\left(J^{n}\right)$ is not a $Z$-set in $J^{n+1}$. Let $I^{\infty}=\operatorname{dir} \lim \left\{I^{n} ; i_{n}\right\}$, $J^{\infty}=\operatorname{dir} \lim \left\{J^{n} ; j_{n}\right\}$.

LEMMA 1. There is a homeomorphism $h: J^{\infty} \rightarrow I^{\infty}$ such that $h / J^{n}$ is piecewise linear, $n \geqslant 1$, and

$$
h\left(J^{n}\right)=\left\{\left(x_{1}, \ldots, x_{n+1}\right) \in I^{n+1} \mid x_{i}=0 \text { some } i, 2 \leqslant i \leqslant n+1\right\} .
$$

Proof. Let

$$
J_{+}^{n}=\left\{\left(x_{1}, \ldots, x_{n}\right) \in J^{n} \mid x_{n} \geqslant 0\right\}, J_{-}^{n}=\left\{\left(x_{1}, \ldots, x_{n}\right) \in J^{n} \mid x_{n} \leqslant 0\right\} .
$$

Presented to the Society, November 9, 1974; received by the editors September 6, 1974 and, in revised form, November 11, 1974.

AMS (MOS) subject classifications (1970). Primary 46A05, 54C55, 57A17, 57C05; Secondary $57 \mathrm{~A} 20$.

Key words and phrases. Hilbert cube, direct limit, absolute (neighborhood) retract, bounded weak-* topology.

${ }^{1}$ Supported in part by NSF Grant GP-43772. 
Note $\left[8,3.15\right.$, p. 36] that any p.l. (piecewise linear) homeomorphism $J^{n}$ $\rightarrow \partial I^{n+1}$ extends to a p.l. homeomorphism $J_{+}^{n+1} \rightarrow I^{n+1}$.

Inductively, we define p.l. embeddings $h_{n}: J^{n} \rightarrow I^{n+1}$ such that

$$
h_{n}\left(J^{n}\right)=\left\{\left(x_{1}, \ldots, x_{n+1}\right) \in I^{n+1} \mid x_{i}=0 \text {, some } i, 2 \leqslant i \leqslant n+1\right\},
$$

and such that $h_{n+1}$ is an extension of $h_{n}$. Define $h_{1}: J^{1} \rightarrow I^{2}$ by $h_{1}\left(x_{1}\right)$ $=\left(\left(x_{1}+1\right) / 2,0\right)$. Having defined $h_{n}$, let $h_{n}^{\prime}: J_{+}^{n+1} \rightarrow I^{n+1}$ be a p.l. homeomorphism extending $h_{n}$. Define $h_{n+1}$ by

$$
h_{n+1}\left(x_{1}, \ldots, x_{n+1}\right) \begin{cases}\left(h_{n}^{\prime}\left(x_{1}, \ldots, x_{n+1}\right), 0\right) & \text { on } J_{+}^{n+1}, \\ \left(h_{n}\left(x_{1}, \ldots, x_{n}\right),-x_{n+1}\right) & \text { on } J_{-}^{n+1} .\end{cases}
$$

The desired homeomorphism $h: J^{\infty} \rightarrow I^{\infty}$ can now be defined by $h / J^{n}=h_{n}$.

Let $B_{n}=[-n, n]^{n}, C_{n}=[0, n]^{n}$. Define $\phi_{n}^{k}: B_{n}^{k} \rightarrow B_{n}^{k+1}, \psi_{n}^{k}: C_{n}^{k} \rightarrow C_{n}^{k+1}$ by $\phi_{n}^{k}\left(x_{1}, \ldots, x_{k}\right)=\left(x_{1}, \ldots, x_{k}, 0\right), x_{i} \in B_{n}, \psi_{n}^{k}\left(x_{1}, \ldots, x_{k}\right)=\left(x_{1}, \ldots, x_{k}, 0\right)$, $x_{i} \in C_{n}$. Let $B_{n}^{\infty}=\operatorname{dir} \lim _{k}\left\{B_{n}^{k} ; \phi_{n}^{k}\right\}, C_{n}^{\infty}=\operatorname{dir} \lim _{k}\left\{C_{n}^{k} ; \psi_{n}^{k}\right\}$.

LEMMA 2. For each $n \geqslant 1$ there is a homeomorphism $g_{n}: C_{n}^{\infty} \times Q \rightarrow Q^{\infty}$ such that $g_{n}\left(C_{n}^{k} \times Q\right)=Q^{k}$, all $k \geqslant 1$.

Proof. Fix $n$. Let $\delta_{k}=\left(\psi_{n}^{k} \times\right.$ id $): C_{n}^{k} \times Q \rightarrow C_{n}^{k+1} \times Q, k \geqslant 1$. Let $\epsilon_{k}$ : $C_{n}^{k} \times Q \rightarrow Q^{k}, k \geqslant 1$, be any sequence of homeomorphisms such that $\epsilon_{k+1} \delta_{k}\left(C_{n}^{k} \times Q\right)$ is a $Z$-set in $Q^{k+1}$. Consider

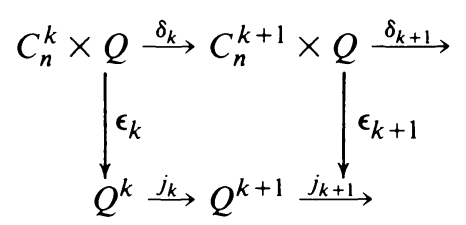

where each $j_{k}: Q^{k} \rightarrow Q^{k+1}$ is the inclusion. Both $A_{2}=\epsilon_{2} \delta_{1}\left(C_{n}^{1} \times Q\right)$ and $B_{2}=j_{1} \epsilon_{1}\left(C_{n}^{1} \times Q\right)$ are $Z$-sets in $Q^{2}$. By a theorem of Anderson [1, Corollary 10.3] we can extend $j_{1} \epsilon_{1} \delta_{1}^{-1} \epsilon_{2}^{-1}: A_{2} \rightarrow B_{2}$ to a homeomorphism $G_{2}: Q^{2} \rightarrow Q^{2}$. By replacing $\epsilon_{2}$ with $G_{2} \epsilon_{2}$, we may thus assume $j_{1} \epsilon_{1}=\epsilon_{2} \delta_{1}$. Doing this for each $\epsilon_{k}, k \geqslant 2$, we obtain a homeomorphism $\epsilon: \operatorname{dir}_{k}\left\{\lim _{n}^{k} \times Q ; \delta_{k}\right\} \rightarrow Q^{\infty}$. Arguing as in the proof of Theorem 4.4, p. 263 of [2], we see that $C_{n}^{\infty} \times Q$ $=\operatorname{dir} \lim _{k}\left\{C_{n}^{k} \times Q ; \delta_{k}\right\}$, and we can take $g_{n}=\epsilon$.

LEMma 3. Let $X, Y$ be subsets of $Q^{\infty}$ such that there exist arbitrarily large integers $r$ and such that $X \cap Q^{r}$ is a Z-set in $Q^{r}$ and $Y \cap Q^{s}$ is a Z-set in $Q^{s}$. Then any homeomorphism $f: X \rightarrow Y$ extends to a homeomorphism $f^{\prime}: Q^{\infty}$ $\rightarrow Q^{\infty}$.

Proof. For $n \geqslant 1$ and $A \subset Q^{\infty}$, let $A_{n}=A \cap Q^{n}$.

Inductively, suppose we have integers $r_{1}, \ldots, r_{n}, s_{1}, \ldots, s_{n}$ and homeomorphisms $f_{1}, \ldots, f_{n}$ such that $f_{i}: Q^{r_{i}} \rightarrow Q^{s_{i}}, i=1, \ldots, n$, and (a) $f_{i} / X_{r_{i}}=f / X_{r_{i}}$, $i=1, \ldots, n$, (b) $f_{i}^{-1}\left(Y_{s_{i-1}}\right) \subset X_{r_{i}}, i=2, \ldots, n$, (c) $F_{i}\left(Q^{r_{i-1}}\right) \cap Y_{s_{i}}=f\left(X_{r_{i-1}}\right)$, $i=2, \ldots, n$, and

(d) $f_{i} /\left[Q^{r_{i-2}} \cup f_{i-1}^{-1}\left(Q^{s_{i-2}}\right)\right]=f_{i-1} /\left[Q^{r_{i-2}} \cup f_{i-1}^{-1}\left(Q^{s_{i-2}}\right)\right], \quad i=3, \ldots, n$. 
(We assume $n \geqslant 3$. The constructions of $f_{1}, f_{2}, f_{3}$ should be clear from the general inductive step.) Choose $r_{n+1}>r_{n}$ such that $f^{-1}\left(Y_{s_{n}}\right) \subset X_{r_{n+1}}$ and $X_{r_{n+1}}$ is a $Z$-set in $Q^{r_{n+1}}$. Then choose $s_{n+1}>s_{n}$ such that $f\left(X_{r_{n+1}}\right) \subset Y_{s_{n+1}}$ and $Y_{s_{n+1}}$ is a $Z$-set in $Q^{s_{n+1}}$. Let $B=Q^{s_{n}} \cup Y_{s_{n+1}}, C=Q^{s_{n-1}} \cup f_{n}\left(Q^{r_{n-1}}\right) \cup f_{n}\left(X_{r_{n}}\right)$. Note that $C$ is a closed subset of $B$, and $B$ is a $Z$-set in $Q^{s_{n+1}}$. Let

$$
\begin{aligned}
& D=\left\{x=\left\{x_{i}\right\} \in Q \mid x_{1}=x_{2}=1\right\}, \\
& E=\left\{x \in Q \mid x_{1}=1\right\} \cup\left\{x \in Q \mid x_{2}=1\right\} .
\end{aligned}
$$

Let $h: Q^{s_{n+1}} \rightarrow Q$ be a homeomorphism such that $h(B) \subset D[1$, Theorem 8.1 and Corollary 10.3]. Let $\phi: D \rightarrow\left[0, \frac{1}{2}\right]$ be a continuous map such that $\phi^{-1}(0)=h(C)$. Given $d \in D$, define $\delta_{d}:[0,2] \rightarrow[0,2]$ by

$$
\delta_{d}(t)= \begin{cases}t(1+\phi(d)), & t \in[0,1], \\ 1+\phi(d)+(t-1)(1-\phi(d)), & t \in[1,2] .\end{cases}
$$

Define $\rho: E \rightarrow D$ by $\rho(x)=\left(1,1, x_{3}, x_{4}, \ldots\right)$. Define $g: E \rightarrow E$ by

$$
g(x)=\left\{\begin{array}{l}
\left(\min \left\{1, \delta_{\rho(x)}\left(x_{1}\right)\right\}, 1-\max \left\{0, \delta_{\rho(x)}\left(x_{1}\right)-1\right\}, x_{3}, x_{4}, \ldots\right) \quad \text { if } x_{2}=1, \\
\left(1,2-\delta_{\rho(x)}\left(2-x_{2}\right), x_{3}, x_{4}, \ldots\right) \text { if } x_{1}=1
\end{array}\right.
$$

Then $g$ is a homeomorphism "sliding points around the corner $D$ ", and for $x \in D, g(x) \in D \Leftrightarrow x \in h(C) \Leftrightarrow g(x)=x$. Since $E$ is a $Z$-set in $Q$, we can extend $g$ to a homeomorphism $g^{\prime}: Q \rightarrow Q$. Let $f_{n}^{\prime}=h^{-1} g^{\prime} h f_{n}: Q^{r_{n}} \rightarrow Q^{s_{n+1}}$. Then $f_{n}^{\prime}$ is an embedding such that (i) $f_{n}^{\prime}$ agrees with $f_{n}$ on $f_{n}^{-1}\left(Q^{s_{n-1}}\right) \cup Q^{r_{n-1}}$ $\cup X_{r_{n}}$, (ii) $f_{n}^{\prime}\left(Q^{r_{n}}\right)$ is a $Z$-set in $Q^{s_{n+1}}$, and (iii) $f_{n}^{\prime}\left(Q^{r_{n}}\right) \cap Y_{s_{n+1}}=f\left(X_{r_{n}}\right)$. To prove (iii), let

$$
y=f_{n}^{\prime}(x)=\left(h^{-1} g^{\prime}\right)\left(h f_{n}\right)(x) \in f_{n}^{\prime}\left(Q^{r_{n}}\right) \cap Y_{s_{n+1}} .
$$

Then $h f_{n}(x) \in D$ and $g^{\prime} h f_{n}(x)=g h f_{n}(x) \in h\left(Y_{s_{n+1}}\right)$ imply that $g h f_{n}(x)$ $=h f_{n}(x)$ so that $h\left(f_{n}(x)\right) \in h(C)$. Thus,

$$
\begin{aligned}
f_{n}^{\prime}(x) & =f_{n}(x) \in Y_{s_{n+1}} \cap C \\
& =Y_{s_{n-1}} \cup\left[Y_{s_{n}} \cap f_{n}\left(Q^{r_{n-1}}\right)\right] \cup\left[Y_{s_{n+1}} \cap f_{n}\left(X_{r_{n}}\right)\right] \subset f_{n}\left(X_{r_{n}}\right)
\end{aligned}
$$

by induction hypotheses (b) and (c). The other containment is clear. Because of (i) and (iii) above we may extend $f_{n}^{\prime}$ to an embedding $f_{n}^{\prime \prime}: Q^{r_{n}} \cup X_{r_{n+1}}$ $\rightarrow Q^{s_{n+1}}$ by defining $f_{n}^{\prime \prime} / X_{r_{n+1}}=f / X_{r_{n+1}}$. Since the image of $f_{n}^{\prime \prime}$ is a $Z$-set in $Q^{s_{n+1}}$, we may extend [1, Corollary 10.3] $f_{n}^{\prime \prime \prime}$ to a homeomorphism $f_{n+1}: Q^{r_{n+1}} \rightarrow Q^{s_{n+1}}$ satisfying our induction hypotheses.

By induction we obtain $f_{n}$, all $n$. The desired homeomorphism $f^{\prime}: Q^{\infty} \rightarrow Q^{\infty}$ can now be defined by $f^{\prime} / Q^{r_{n}}=f_{n+1} / Q^{r_{n}}$.

THEOREM 4. $R^{\infty} \times Q \cong Q^{\infty}$.

Proof. Fix $n \geqslant 1$. Define $\beta_{n}: B_{n}^{\infty} \rightarrow J^{\infty}, \gamma_{n}: C_{n}^{\infty} \rightarrow I^{\infty}$ by

$$
\beta_{n}\left(x_{1}, x_{2}, \ldots\right)=(1 / n)\left(x_{1_{1}}, \ldots, x_{1_{n}}, x_{2_{1}}, \ldots, x_{2_{n}}, \ldots\right)
$$


(here $\left.x_{i}=\left(x_{i_{1}}, \ldots, x_{i_{n}}\right) \in B_{n}\right)$,

$$
\gamma_{n}\left(x_{1}, x_{2}, \ldots\right)=(1 / n)\left(x_{1_{1}}, \ldots, x_{1_{n}}, x_{2_{1}}, \ldots, x_{2_{n}}, \ldots\right) \text {. }
$$

Define $\alpha_{n}: B_{n}^{\infty} \rightarrow B_{n+1}^{\infty}$ by

$$
\left(x_{1_{1}}, \ldots, x_{1_{n}}, x_{2_{1}}, \ldots, x_{2_{n}}, \ldots\right) \rightarrow\left(x_{1_{1}}, \ldots, x_{1_{n}}, 0, x_{2_{1}}, \ldots, x_{2_{n}}, 0, \ldots\right) .
$$

Let $h_{n}=\left(\gamma_{n}^{-1} h \beta_{n} \times\right.$ id $): B_{n}^{\infty} \times Q \rightarrow C_{n}^{\infty} \times Q$, where $h$ is the homeomorphism of Lemma 1. Clearly $h_{n}$ is a homeomorphism. Consider

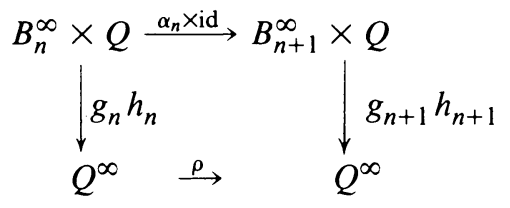

Here $g_{n}$ is the homeomorphism of Lemma 2, and $\rho$ is defined by $\rho\left(x_{1}, x_{2}, \ldots\right)$ $=\left(x_{1}, 0, x_{2}, 0, \ldots\right), x_{i} \in Q$. Let

$$
X=g_{n+1} h_{n+1}\left(\alpha_{n} \times \mathrm{id}\right)\left(B_{n}^{\infty} \times Q\right), \quad Y=\rho g_{n} h_{n}\left(B_{n}^{\infty} \times Q\right) .
$$

Clearly $Y \cap Q^{k}$ is a $Z$-set in $Q^{k}$, all large $k$. We now show this is also true for $X$ :

$$
\begin{aligned}
X \cap Q^{k} & =g_{n+1}\left(\left[\gamma_{n+1}^{-1} h \beta_{n+1} \alpha_{n}\left(B_{n}^{\infty}\right) \cap C_{n+1}^{k}\right] \times Q\right) \\
& =g_{n+1}\left[\left(\gamma_{n+1}^{-1} h\left(J^{n} \times 0 \times J^{n} \times 0 \times \cdots\right) \cap \gamma_{n+1}^{-1}\left(I^{k n+k}\right)\right) \times Q\right]
\end{aligned}
$$

which is a closed subset of $g_{n+1}\left[\gamma_{n+1}^{-1}\left(h\left(J^{k n+k-1}\right)\right) \times Q\right]$ since $h\left(J^{r}\right) \supset I^{r}$. Since $h\left(J^{r}\right)$ is a $Z$-set in $I^{r+1}$, it follows that $X \cap Q^{k}$ is a $Z$-set in $g_{n+1}\left[\gamma_{n+1}^{-1}\left(I^{k n+k}\right)\right.$ $\times Q]=Q^{k}$. Thus, applying our Lemma 3 as Corollary 10.3 of [1] was applied in the proof of Lemma 2, we obtain a homeomorphism

$$
\operatorname{dir} \lim _{n}\left\{B_{n}^{\infty} \times Q ; \alpha_{n} \times \text { id }\right\} \rightarrow \operatorname{dir} \lim \left\{Q^{\infty} ; \rho\right\}
$$

We finish the proof by showing that the first direct limit (above) is $R^{\infty} \times Q$, the latter $Q^{\infty}$. A proof analogous to the proof of Theorem 4.4, p. 263 of [2] shows that

$$
\operatorname{dir} \lim _{n}\left\{B_{n}^{\infty} \times Q ; \alpha_{n} \times \mathrm{id}\right\} \cong\left(\operatorname{dir}_{n} \lim _{n}\left\{B_{n}^{\infty} ; \alpha_{n}\right\}\right) \times Q .
$$

Define $\mu_{n}: B_{n}^{\infty} \rightarrow\left(R^{\infty}\right)^{\infty}$ by

$$
\begin{aligned}
\mu_{n}\left(\left(x_{1_{1}}, \ldots, x_{1_{n}}\right),\left(x_{2_{1}}, \ldots, x_{2_{n}}\right), \ldots\right) \\
\quad=\left(\left(x_{1_{1}}, \ldots, x_{1_{n}}, 0,0, \ldots\right),\left(x_{2_{1}}, \ldots, x_{2_{n}}, 0,0, \ldots\right), \ldots\right) .
\end{aligned}
$$

Define $\mu: \operatorname{dir} \lim _{n}\left\{B_{n}^{\infty} ; \alpha_{n}\right\} \rightarrow\left(R^{\infty}\right)^{\infty}$ by $\mu / B_{n}^{\infty}=\mu_{n}$. By Proposition II-1(a) and Corollary III-3 of [4], sets of the form

$$
\left[\left(\theta_{1_{1}} \times \theta_{1_{2}} \times \cdots\right) \times\left(\theta_{2_{1}} \times \theta_{2_{2}} \times \cdots\right) \times \cdots\right] \cap\left(R^{\infty}\right)^{\infty},
$$


$\vartheta_{i_{j}}$ open in $R$, are a basis for $\left(R^{\infty}\right)^{\infty}$. It follows easily that $\mu$ is continuous. By Corollary III-2 of [4], $\mu^{-1}$ is continuous if it is continuous on each $\left(R^{k}\right)^{n}$ (and hence if continuous on each $\left.\left(B_{k}\right)^{n}\right)$, and this is clear. Finally [4, Corollary III-3], $\left(R^{\infty}\right)^{\infty} \cong R^{\infty}$ so that, indeed,

$$
\operatorname{dir} \lim _{n}\left\{B_{n}^{\infty} \times Q ; \alpha_{n} \times \mathrm{id}\right\} \cong R^{\infty} \times Q .
$$

Define $\tau_{n}:\left(Q^{\infty}\right)^{n} \rightarrow Q^{\infty}$ by

$$
\tau_{n}\left(\left\{x_{1_{j}}\right\}, \ldots,\left\{x_{n_{j}}\right\}\right)=\left(x_{1_{1}}, x_{2_{1}}, \ldots, x_{n_{1}}, x_{1_{2}}, \ldots, x_{n_{2}}, \ldots\right) .
$$

Consider

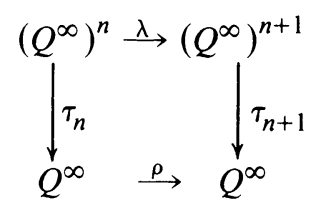

where $\lambda$ is inclusion. Then $\tau_{n+1} \lambda\left[\left(Q^{\infty}\right)^{n}\right]$ and $\rho \tau_{n}\left[\left(Q^{\infty}\right)^{n}\right]$ satisfy the hypotheses of Lemma 3. As before, by applying our Lemma 3, we obtain a homeomorphism of direct limits, $\left(Q^{\infty}\right)^{\infty} \cong \operatorname{dir} \lim \left\{Q^{\infty} ; \rho\right\}$. Since $\left(Q^{\infty}\right)^{\infty} \cong Q^{\infty}[4$, Corollary III-3], this completes the proof of Theorem 4.

Corollary 5. Let $U, V$ be open subsets of $R^{\infty}$. Then $U$ and $V$ have the same homotopy type iff $U \times Q$ is homeomorphic to $V \times Q$.

Proof. By Theorem $4, U \times Q$ and $V \times Q$ are open subsets of $Q^{\infty}$, and as such they are homeomorphic iff they have the same homotopy type [5, Theorem II-1 and Corollary II-12].

Lemma 6. Let $X$ be a locally compact, separable ANR (metric). Then $X$ embeds as a closed subset of $Q^{\infty}$.

Proof. Since $X$ is Lindelöf and locally compact, we can write $X=\bigcup\left\{X_{i} \mid i\right.$ $=1,2, \ldots\}$ where each $X_{i}$ is a compact, metric subspace and $X_{i} \subset$ int $X_{i+1}$. Let $h_{1}: X_{1} \rightarrow Q^{1}$ be any (closed) embedding [2, p. 195]. Suppose, inductively, that we have embeddings $h_{i}: X_{i} \rightarrow Q^{i}, i=1, \ldots, n$, such that $h_{i} / X_{i-1}=h_{i-1}$, $i>2$, and $h_{n}\left(X_{n}\right) \cap Q^{i}=h_{n}\left(X_{i}\right), i<n$. Let $g_{n+1}: X_{n+1} \rightarrow Q^{n} \subset Q^{n+1}$ be any embedding. By $\left[1\right.$, Corollary 10.3] the induced homeomorphism $g_{n+1}\left(X_{n}\right)$ $\rightarrow h_{n}\left(X_{n}\right)$ extends to a homeomorphism $g: Q^{n+1} \rightarrow Q^{n+1}$. Then $f_{n+1}=g g_{n+1}$ : $X_{n+1} \rightarrow Q^{n+1}$ is an embedding such that $f_{n+1} / X_{n}=h_{n}$. Proceeding as in the proof of Lemma 3 with $B=Q^{n} \cup f_{n+1}\left(X_{n+1}\right)$ and $C=f_{n+1}\left(X_{n}\right)$, we can now adjust $f_{n+1}$ to obtain an embedding $h_{n+1}: X_{n+1} \rightarrow Q^{n+1}$ such that $h_{n+1} / X_{n}$ $=h_{n}$ and $h_{n+1}\left(X_{n+1}\right) \cap Q^{n}=h_{n+1}\left(X_{n}\right)$. In this way we obtain $h_{n}$, all $n$. The desired closed embedding $h: X \rightarrow Q^{\infty}$ can now be defined by $h / X_{n}=h_{n}$.

THEOREM 7. (a) If $X$ is a locally compact, separable AR (metric), then $X \times Q \times R^{\infty} \cong Q \times R^{\infty}$. (b) If $X$ is a locally compact, separable ANR (metric), then $X \times Q \times R^{\infty}$ embeds as an open subset of $Q \times R^{\infty}$.

PROof. (If it were known that $Q^{\infty}$ is an AR for perfectly normal spaces, a proof analogous to the proof of Theorem 2 of [6] would work here.) By [4, 
Theorem II-6] we may identify $Q \times R^{\infty} \cong Q^{\infty}$ with $L$, by which we denote a separable, infinite-dimensional Hilbert space with its bounded weak topology. Since $L$ is a topological vector space [3, Corollary 5, p. 428] and $L^{\infty} \cong L[4$, Corollary III-3], the proof of Henderson [6] shows that if $X$ is a retract of $L$, then $X \times L \cong L$.

Now suppose $X$ is a locally compact, separable ANR (metric). By Lemma 6 we may regard $X$ as a closed subspace of $L$. By [7, Theorem 4.1, p. 86] $X$ is also an ANR (perfectly normal). By Proposition III-1 of [5] $L$ is normal, and the proof of that same proposition shows that each open subset of $L$ is an $F_{\sigma}$ so that $L$ is perfectly normal. Thus, there is a neighborhood $U$ of $X$ in $L$ and a retraction $r: U \rightarrow X$. Choose open sets $V$ and $W$ in $L$ such that $X \subset V \subset \bar{V}$ $\subset W \subset \bar{W} \subset U$, and then a continuous map $\phi: L \rightarrow[0,1]$ such that $\phi^{-1}(0)$ $=X$ and $\phi /(L \backslash V)=1$. Let

$$
Y_{1}=\{(x, t) \in \bar{W} \times R \mid t \geqslant \phi(x)\}, \quad Y_{2}=\{(x, t) \in L \times R \mid t \leqslant \phi(x)\},
$$

and $Y_{3}=Y_{1} \cap Y_{2}$. Define $r_{1}: Y_{1} \rightarrow X \times[0, \infty)$ and $r_{2}: Y_{3} \cup(L \times\{0\}) \rightarrow L$ $\times\{0\}$ by $r_{1}(x, t)=(r(x), t-\phi(x)), r_{2} /(L \times\{0\})=\mathrm{id}$, and $r_{2} / Y_{3}=r_{1}$. (This is where $\phi^{-1}(0)=X$ is used.) It follows easily from the Banach-Dieudonné theorem [3, p. 427] that $L \times R \cong L$. Thus, we may extend $r_{2}$ to a continuous map $r_{2}^{\prime}: L \times R \rightarrow L \times\{0\} \subset L \times R$ [5, Proposition III-3]. Let $X^{\prime}=(X$ $\times[0, \infty)) \cup(L \times\{0\})$. Define $\lambda: Y_{1} \cup Y_{2} \rightarrow X^{\prime}$ by $\lambda / Y_{1}=r_{1}$ and $\lambda / Y_{2}=r_{2}^{\prime}$. Then $\lambda$ is a retraction. Since $Y_{1} \cup Y_{2} \supset(W \times R) \cup[(L \backslash \bar{V}) \times(-\infty, 1)]$, a neighborhood of $X^{\prime}$, we thus have that $X^{\prime}$ is a neighborhood retract of $L \times R$. Also, $X^{\prime}$ is contractible, and the same proof that shows that a contractible ANR is an AR now shows that $X^{\prime}$ is a retract of $L \times R \cong L$. Thus, by Henderson's theorem, $X^{\prime} \times L \cong L$ so that $X \times L \cong X \times(0, \infty) \times L$ is open in $L$. This establishes (b). Since contractible open subsets of $Q^{\infty}$ are homeomorphic to $Q^{\infty}$ [5, Corollary II-12], (a) now follows immediately.

\section{REFERENCES}

1. R. D. Anderson, On topological infinite deficiency, Michigan Math. J. 14 (1967), 365-383. MR 35 \#4893.

2. J. Dugundji, Topology, Allyn and Bacon, Boston, Mass., 1966. MR 33\#1824.

3. N. Dundford and J. T. Schwartz, Linear operators. I: General theory, Pure and Appl. Math., vol. 7, Interscience, New York, 1964.

4. R. E. Heisey, Contracting spaces of maps on the countable direct limit of a space, Trans. Amer. Math. Soc. 192 (1974), 389-411.

5. - Manifolds modelled on $R^{\infty}$ or bounded weak-* topologies, Trans. Amer. Math. Soc. 206 (1975), 295-312.

6. D. W. Henderson, A simplicial complex whose product with any ANR is a simplicial complex, General topology and Appl. 3 (1973), 81-83. MR 47 \#208.

7. S. -T. Hu, Theory of retracts, Wayne State Univ. Press, Detroit, Mich., 1965. MR 31 \#6202.

8. C. P. Rourke and B. J. Sanderson, Introduction to piecewise-linear topology, Ergebnisse der Mathematik und ihrer Grenzgebiete, Band 69, Springer-Verlag, New York, 1972.

Department of Mathematics, Vanderbilt University, Nashville, Tennessee 37235 